J. EDUCATIONAL COMPUTING RESEARCH, Vol. 29(2) 229-252, 2003

\title{
SEARCHING FOR INFORMATION ON-LINE AND OFF-LINE: GENDER DIFFERENCES AMONG MIDDLE SCHOOL STUDENTS*
}

\author{
MARGUERITE ROY \\ ROGER TAYLOR \\ MICHELENE T. H. CHI \\ University of Pittsburgh
}

\begin{abstract}
There has been a national call for increased use of computers and technology in schools. Currently, however, little is known about how students use and learn from these new technologies. This study examines how students search for, browse, and learn specific information when performing an on-line (Web) versus an off-line (Library) search. Twenty-eight eighth grade students between the ages of 13 and 14 were randomly assigned to one of the two search conditions (Web or Library), each balanced by gender. All participants significantly improved their knowledge of the target question ("How do mosquitoes find their prey?") from pre-test to post-test. The Web was superior to the Library in supporting students search for target-specific information. There was an interaction in which boys in the Web condition learned more target-specific and target-related information than the girls. Several related findings are explored which may help explain this result.
\end{abstract}

\section{INTRODUCTION}

There has been a call for increased use of computers and technology in the classroom. Indeed, one of the seven priorities recently set out by the U.S. Department of

*The authors are grateful for the support from the Spencer Foundation granted to Michelene T. H. Chi. 
Education (1997) included the following goal: "All students should be able to have their classroom connected to the Internet by the year 2000 and be technologically literate." In response to such recommendations, schools have been spending increasingly large proportions of their limited funds to acquire computers, software, and related resources (Pelavin Research Institute, 1997). The question of whether this kind of investment will have any effective educational payoffs needs to be addressed.

The basic assumption that underlies such recommendations is that incorporating more technology into the classroom will be associated with positive changes in terms of increases in student learning opportunities and outcomes. Currently, however, such general claims have not been substantiated in the education literature. In fact, little is known about how students are able to make use of and learn from these new technologies. For example, there is little research investigating how students are able to search for and use information that they find on the World Wide Web (Web).

Many positive claims have been made with regard to the educational potential of technology in general and the Web in particular (Owston, 1997). Some have pointed out that advances in information technology, like the rapid growth and use of the Web, have the potential to influence both the way that users seek and make use of information (Bilal, 2000; Nahl, 1998). To date, however, only a few studies have examined student's use of the Web. In general, such studies have either documented faults or shortcomings in students' search behavior that may explain why they fail to find "useful" information (Bilal, 1999; Schacter, Chung, \& Dorr, 1998), or have described student's attitudes toward using on-line information retrieval methods like the Web (Lange \& Beheshti, 2000). For example, children tend to browse and react to information, rather than to plan and employ systematic analytic search strategies when seeking specific information on the Internet (Schacter et al., 1998). Also, unlike adults, children tend to believe that all information they encounter on the Internet is true. In addition, they tend to make spelling and typographical errors (Fidel, Davies, Douglass, Holder, Hopkins, Kushner, Miyagishima, \& Toney, 1999).

While these are important issues, what is also needed is research which links patterns of student search behavior to learning outcomes across different search conditions (i.e., on-line verses off-line). Do students learn differently under these two search conditions? Do the ways that they search for information on the Web differ from the ways that they search for information in their local libraries? Do students find different amounts and types of information when they perform an on-line Web search as opposed to an off-line library search? Do students treat information that they "find" when using the Web differently than that found by more traditional off-line methods? These are some of the questions that must be addressed to substantiate the claims that are currently being made about the potential benefits of increased use of computers and technology in the classroom in general, and the Web in particular. 
Associated with the call for increased use of computers in the classrooms is the issue of whether all students participate equally and receive similar benefits from such changes. One potential barrier to embracing technology on a broad basis in particular is inequality of benefits for girls and boys (Light, Littleton, Bale, Joiner, \& Messer, 2000). The issue of gender differences in relation to computer-based learning and technology use has generally centered around findings that boys tend to demonstrate more positive attitudes (Kadijevich, 2000; Schumacher \& Morahan-Martin, 2001; Todman \& Dick, 1993) and learning outcomes than girls (Light et al., 2000). Such differences have sometimes been attributed to a propensity for boys to have greater experience with using computers (Mitra et al., 2000; Nachmais, Mioduser, \& Shemla, 2001).

The effects of such gender differences have important and real implications. For example, Camp (1997) has reported a disturbing trend whereby the proportion of women who select science or engineering as their major at the undergraduate level has rapidly decreased over the past few years. It is suggested that this trend reflects women's growing disenfranchised attitudes toward science and technology. However, it is not clear what factors are responsible for such attitudes. Nor is it clear whether incorporating more technology into the classroom will widen or close the gender gap.

The primary purpose of the current study is to compare the quantity and quality of the information retrieved and the associated knowledge gained by students conducting either a set of on-line Web-based searches or more traditional off-line library-based searches for specific targeted information. In order to assess the possibility that students learn information that is related but not specific to the target we are interested in assessing both target-specific gains as well as knowledge gains which are target-related (i.e., incidental learning gains). It's important to assess target-related knowledge gains in addition to target-specific learning since such gains would give us a sense of how much relevant information students might encode while they are browsing. In addition, we are interested in ascertaining whether any differences associated with these two search conditions are affected by gender. In particular, we want to evaluate how students gather, learn, and use information. The traditional method of searching for academically related information is to use the physical resources provided by one's local school Library. A newer, and potentially more effective method of searching for academically related information is to use the electronic resources provided by Web browsers and the Internet. No previous studies have examined how these two approaches compare in terms of the search patterns used, the quantity and quality of information retrieved, and knowledge gained.

Without making a specific prediction, the goal of this project is to explore and compare the effectiveness of on-line Web searches with that of conventional off-line searches using a Library.

If navigation strategies needed for effective use of hypermedia environments do not correspond to the kind of comprehension and library search skills that 
students are currently familiar with (Goldman, 1996), then it is conceivable that searching for specific information on the Internet maybe a cumbersome task (Andriessen \& Sandberg, 1999), resulting in failures to obtain the appropriate information, either because the information is embedded in a vast amount of irrelevant facts and figures, or the information lacks censorship and authority. If this is indeed the case, then we would expect the answers that students are able to write in response to a specific search prompt to be less complete, coherent, and factually correct when they are based on Web search than those they are able to write based on a Library search. On the other hand, it is equally conceivable that on-line Web searches are faster, richer, and more efficient than traditional Library searches. If this alternative is correct, then we would expect student answers that are based on the results of a Web search to be superior to answers based on a Library search.

\section{METHOD}

\section{Participants}

Twenty-eight eighth grade students from a local Pittsburgh middle school between the ages of 13 and 14 volunteered to participate in this study. An equal number of boys and girls were randomly assigned to search for information to a specific prompt either: 1) on-line using the Web in the school computer laboratory; or 2) off-line in the school Library.

\section{Materials}

\section{Technical Equipment}

For the Web condition, the experiment was performed on a Macintosh G3 computer running Internet Explorer (v 5.1) as the Web browser. The browser was set to use Google as the Web-based search engine. For the Library condition, participants had free access to an IBM/DOS based computer to search the contents of the library using an electronic card catalogue system in addition to the physical contents of the library itself.

\section{Participant Experience}

A pre-experimental questionnaire was designed for students to report their level of experience using the Web and Google to search for information on-line, as well as their experience using the school Library to search for information. The questionnaire included a total of 11 self-report items (see Table 1), consisting of questions such as: "On average, how much time per week do you spend using the Web for your studies?" 


\section{The Target Domain and Assessment of Knowledge}

Mosquito hunting behavior (i.e., how mosquitoes find their prey) was chosen as the target topic for this study for several reasons. First, an informal survey of the students indicated that the topic was of interest and that most students did not readily know the answer to the question. Second, the topic seemed sufficiently open-ended and challenging, but not so much so that the participants would not be able to answer the questions in the given time period. Finally, we determined that both study conditions (i.e., Web and Library) had sufficient available resources to fully answer the question.

The materials used to measure what students learned in this study included both: 1) an open-ended question to assess each student's knowledge of the target topic; and 2) and a set of 13 incidental learning questions. The specific open-ended question asked was "In your own words, please describe at least three ways that mosquitoes find their prey." This question was administered to assess both pre-search and post-search knowledge of the target question. The set of 13 short-response incidental learning questions was used to assess each student's post-search general knowledge of mosquitoes. These questions were designed to assess knowledge of facts that students would likely come across while performing their search, but which were not directly relevant to the target question (e.g., Why are mosquito bites itchy?).

\section{Design and Procedure}

The design used to analyze target learning consisted of a mixed between-within design. The two between subjects factors (Condition: Web, Library; and Gender: Boy, Girl) were crossed with the within subjects factor (Time: Pre-test, Post-test). All remaining analyses were conducted using a two-way between-subjects design with Condition and Gender as the two crossed factors.

A pre-experimental questionnaire was administered to ascertain each student's experience with and attitudes toward performing both on-line Web-based and off-line Library-based information searches.

The experiment itself was conducted in a single session on an individual basis (i.e., each student individually met with the pair of experimenters). Each session lasted one class period (approximately 50 minutes). At the beginning of the session, each student was given up to five minutes to complete the target question. This information was used to assess the student's pre-search knowledge of the target question.

Following the pre-test, each student was given up to 25 minutes to search for answers to the target question. All students were able to take notes during their search. Students in the Web condition were told that they could "bookmark" up to 30 printable pages of resources (by selecting "Add to Favorites" under the Favorites menu). Students in the Library condition were also told that they could "book-mark" (by using Post-Its) up to 30 pages of resources. All students were 
told that they would be permitted to refer back to these pages while answering the target question in the following Post-Test section.

While each student conducted their information search, the two experimenters observed and recorded the participant's search behavior and noted the identity and number of all resources that were examined. The three main categories of behavior that were noted included searching for, scanning, and browsing information.

After completing the search, participants were given 10 minutes to answer the target knowledge question again. For this post-test, participants could use their notes and any of their book-marked resources.

Following the post-test, students were given the unannounced incidental learning test to measure any related knowledge that they might have gained during their target knowledge search. The participants were not allowed to use any of their notes or book-marked material on this assessment.

\section{RESULTS}

\section{Learning Gains}

First, we will present the results of learning gains for both the target-specific knowledge and the target-related outcome measures in order to assess the effects of performing information searches in the two different contexts (Web and Library) for boys and girls.

\section{Learning Target-Specific Knowledge}

To assess students' learning of the target information, each student's Pre- and Post-test answers were scored in terms of the total number of correct idea units written in response to the target question, with a maximum possible score of 18 . At Pre-test, there were no significant differences in target knowledge among the four groups. Thus, all groups started out equal with regard to their prior knowledge of the target question. Overall, there was a significant increase in performance from pre-test $(M=1.43)$ to post-test $(M=4.5)$, regardless of condition or gender $(F(1,24)=39.1, p<0.0005)$. This finding indicates that all students were able to achieve significant gains in target-specific knowledge based on their search performance.

Overall, students in the Web condition demonstrated a larger knowledge gain than those in the Library, although this difference was not statistically significant (compare Figures 1a and 1b). There was no evidence of an overall difference in gains between the two genders. There was, however, an interaction involving condition and gender. Specifically, a posthoc Bonferroni comparison indicated a significant difference in the knowledge gain demonstrated from pre- to posttest between boys and girls in the Web condition $(F(1,12)=5.32, p=0.040)$. As Figure 1a illustrates, boys gained more than girls in this condition, whereas the gains demonstrated in the Library condition were comparable across Gender (see Figure 1b). 


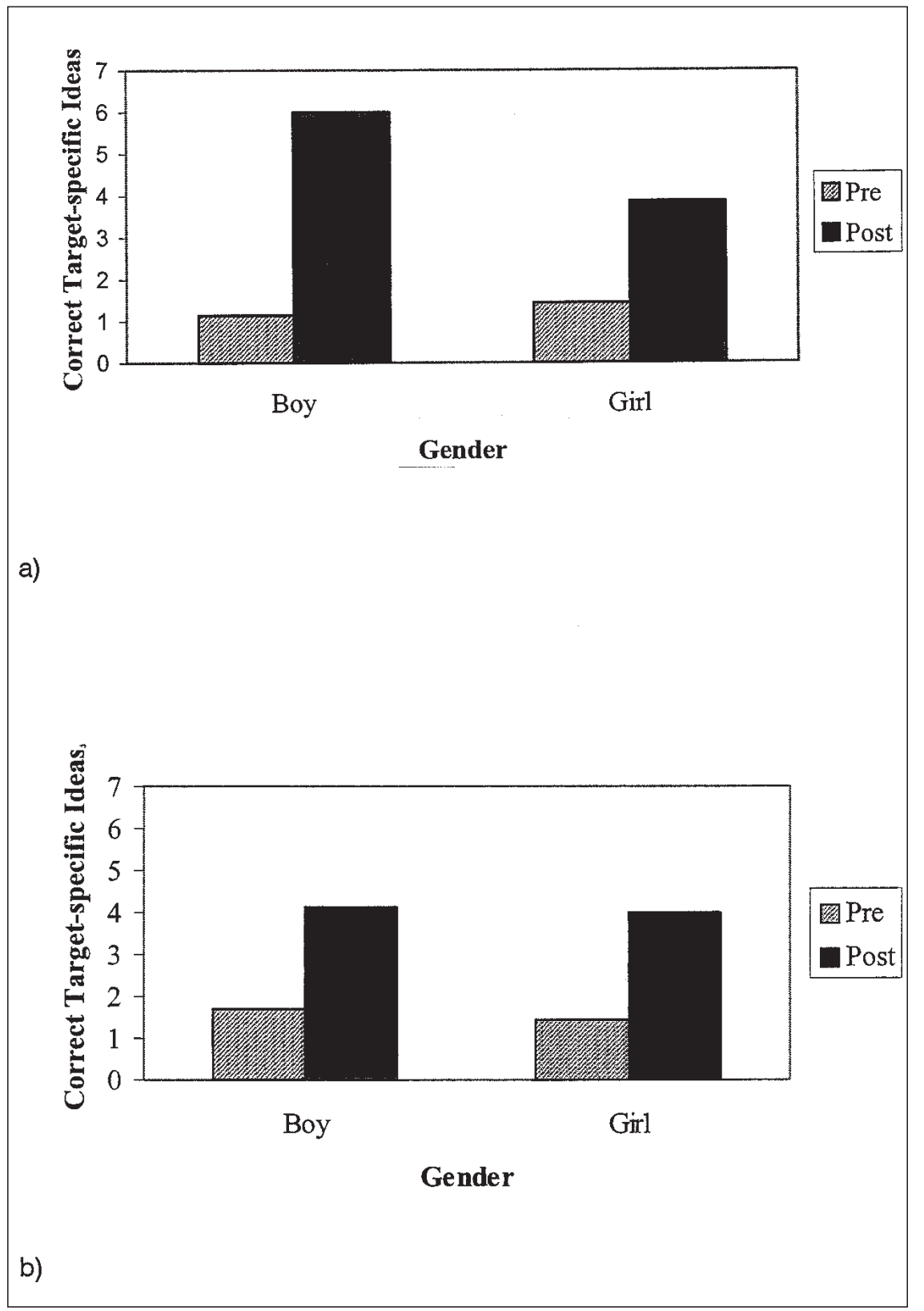

Figure 1. a) Pre and post target-specific knowledge scores by Gender for the Web Condition. b) Pre and post target-specific knowledge scores by Gender for the Library Condition. 


\section{Learning Target-Related Knowledge}

In addition to measuring target-specific learning (i.e., how mosquitoes find their prey), we also assessed students' knowledge of information about mosquitoes in general, information that they were likely to come across while performing the search task (e.g., what kind of diseases mosquitoes carry). ${ }^{1}$ We performed this second assessment at the post-test only in order to investigate possible group differences in students' exposure to and learning of information related to the target information. Each participant's target-related knowledge was measured using the set of 13 short answer questions. With a possible maximum score of 13 , girls $(M=3.11)$ again scored significantly lower than boys on this measure $(M=7.04)$, but only in the Web condition (see Figure 2$)$. The interaction between condition and gender was significant $(F(1,24)=5.91, p=0.023)$. No other effects were significant for this analysis. Thus, as in the case for gains in target-specific knowledge (Figure 1b), gains in target-related knowledge were again comparable between the boys and the girls for the Library condition only.

In sum, boys and girls performed comparably in the Library condition on both target-specific knowledge and target-related knowledge outcome measures. It is only in the Web condition that boys and girls demonstrated distinct performance differences. Specifically, boys in the Web condition acquired the largest target knowledge gains of all groups (see Figure 1a), whereas girls in the Web condition acquired the least amount of incidental or target-related knowledge (see Figure 2).

\section{Exploring the Gender Effect in the Web Condition}

What could have accounted for the tendency of boys to demonstrate different learning gains from girls when searching for information using the Web? To address this question, we performed some additional analyses on the Web group alone. First, we explore whether there were any associated gender differences in the students' pattern of computer or library use, in their academic ability, or in terms of the time they spent performing the task. Next, we explore potential differences in the pattern of students' navigation behavior.

\section{Participants Experience with Using the Web}

Results from the Pre-experimental questionnaire provided us with information about each student's level of experience with and attitudes toward using the Web

\footnotetext{
${ }^{1}$ Twenty percent of the data for the target-specific and target-related measures was randomly drawn to calculate inter-rater reliability between an independent rater and the experimenter who coded the data. The percent agreement between the two raters was 100 percent for the target-specific knowledge measure and 98 percent for the target-related knowledge measure.
} 


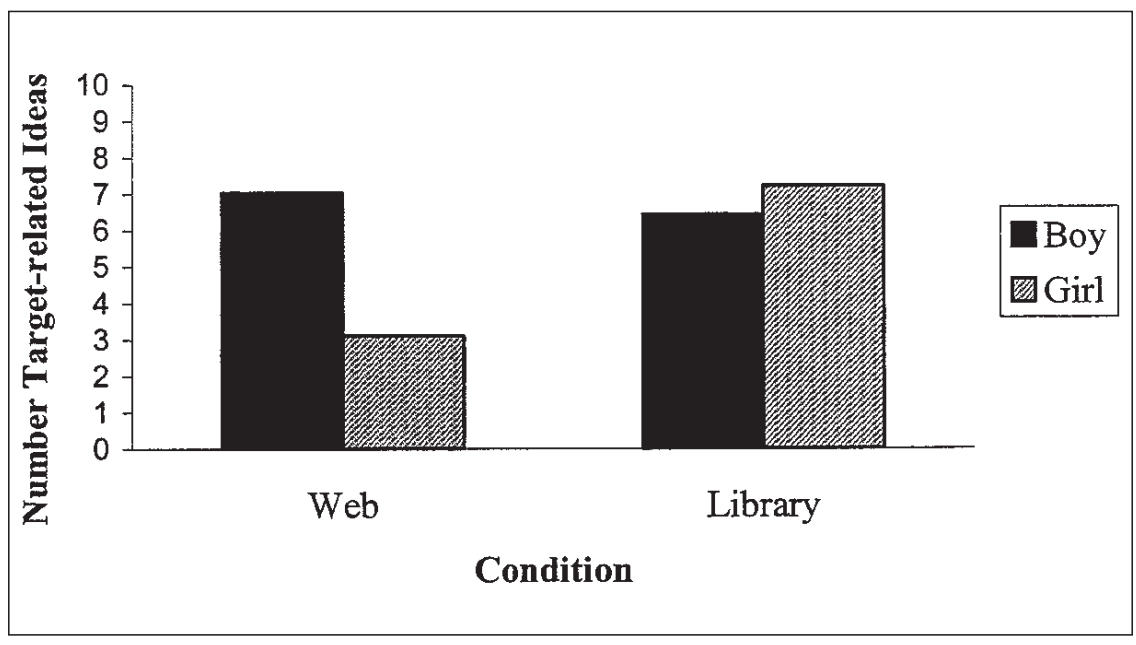

Figure 2. Target-related knowledge scores by Gender and Condition.

and the school Library. The average responses across participants in each group are listed in Table 1. The first question asked respondents whether or not they had access to the Web at home. Twenty-seven of the 28 students report that they do have home access to the Web. Based on responses to this item and the fact that all students had similar access to computers and the Web at school, it is apparent that all groups (including boys and girls in the Web condition) had equal access to the Web. The remaining questions asked students to use a Likert scale to rate their experiences and opinions with regard using both the Web and the school Library to perform information searches. A series of analyses were performed to explore the possibility that there was a pre-existing difference between boys and girls in the Web condition in terms of their experience or attitudes toward using this search method. For example, if boys demonstrate more experience or attribute more usefulness to searching using the Web than girls, then this might explain the observed differences in the learning outcomes.

A series of one-way ANOVAs indicated no significant differences between boys and girls in the Web condition on any of the self-rated items, with one single exception. There was a significant gender difference for question three $(F(1,13)=5.145, p=.005)$. Surprisingly, girls $(M=2.43)$ reported using the Web for schoolwork significantly more often than boys $(M=1.29)$. With this exception, there were no other differences in the way that boy and girl students reported using either the Web or the local Library. Thus, if anything, we would expect more favorable learning outcomes for girls in the Web condition. However, this was not the case. 


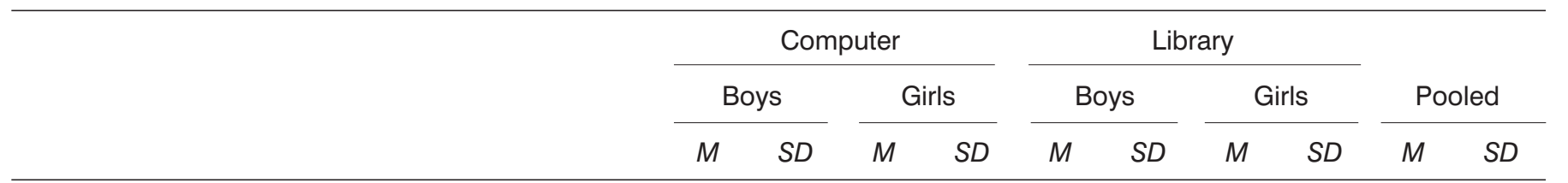

\section{Web questions}

1. WWW access at home.

(number responding yes)

2. Average time per week using the WWW.

(1 > $15 \mathrm{~min}, 2=15 \mathrm{~min}-1 \mathrm{hr}, 3=1-2 \mathrm{hrs}$

$4=2-4 \mathrm{hrs}, 5<4 \mathrm{hrs}$ )

3. Average time per week using WWW for studies.

(1 > $15 \mathrm{~min}, 2=15 \mathrm{~min}-1 \mathrm{hr}, 3=1-2 \mathrm{hrs}$,

$4=2-4 \mathrm{hrs}, 5<4 \mathrm{hrs}$ )

4. Use of Google search engine.

( 1 = never, 2 = > once/month, 3 = monthly, 4 = weekly, 5 = daily)

5. Amount of relevant information found using the WWW.

$(1=$ none, 2 = not enough, 3 = enough, $4=$ more than enough)

6. Rated worth of time used to search the WWW. $(1=$ no, $2=$ somewhat, $3=$ yes $)$

7

3.14

1.29

0.492 .43

2.29

1.38

3

0.58

2.86

2.860

$.43 \quad 0.53$

2.80

.55

$\begin{array}{lllllllll}2.57 & 0.53 & 2.57 & 1.51 & 2.86 & 0.38 & 2.43 & 0.53 & 2.60\end{array}$ 


\section{Library questions}

7. Average time per week using the Library. (1 > $15 \mathrm{~min}, 2=15 \mathrm{~min}-1 \mathrm{hr}, 3=1-2 \mathrm{hrs}$, $4=2-4 \mathrm{hrs}, 5<4 \mathrm{hrs})$

8. Average time per week using the Library for studies.

(1 > 15 min, 2 = 15 min- $1 \mathrm{hr}, 3=1-2 \mathrm{hrs}$

$4=2-4 \mathrm{hrs}, 5<4 \mathrm{hrs}$ )

9. Use of EEC search engine.

$(1=$ never, $2=>$ once $/$ month, $3=$ monthly, $4=$ weekly, 5 = daily)

10. Amount of relevant information found using the Library.

( 1 = none, 2 = not enough, 3 = enough $4=$ more than enough)

11. Worth of time used to search the Library. ( $1=$ no, $2=$ somewhat, $3=$ yes $)$ 


\section{Student Ability}

A subset ${ }^{2}$ of the total sample of students provided their consent to access their academic records. Even though random assignment to conditions was employed, we also analyzed this information to assess the possibility that the gender differences we observed within the Web condition were due to pre-existing differences in student ability. Separate two-way ANOVA were conducted on students: 1) total grade point average for all courses; and 2) grades in Computer class. These analyses revealed no significant effects involving gender. Although these analyses were based on a small sample, the group means for the girls tended to be higher both overall $(M=3.87)$ and in Computer class $(M=4.11)$ then those for boys ( $M=3.27$, and $M=4.0$, respectively). Thus, the learning differences observed above are likely not due to pre-existing differences in student ability.

\section{Time on Task}

Another possible explanation for the observed gender difference was that boys were more successful than girls because they spent differing amounts of time on task. There was no overall difference between boys and girls in terms of the total time they allotted to searching for and browsing information on the Web. In general, each group spent about 18.39 minutes searching. Moreover, an equal number of boys and girls terminated their search activity before reaching the 25 minute maximum time allotted to each participant. Furthermore, there was no significant correlation between time on task and amount or target-specific learning or target-related performance.

\section{Navigation or Information-Seeking Behavior}

What then can account for boy's superiority in the Web condition? One possibility is that boys are more effective somehow in the way they seek information on the Web. In order to investigate the possibility that boys may have employed more effective information navigation skills than girls, we used the field notes taken by the two experimenters during the 25 minute search period as a basis for coding the quantity and quality of each student's navigation behavior as described below.

We were able to differentiate three types of information navigation behavior in order to investigate this possibility. One type of behavior is searching. Database searching consisted of instances where students submitted or entered a set of keywords or phrases into Google to query the database. Once a query is submitted

\footnotetext{
${ }^{2}$ The sub-sample for the analysis of gender differences within the Web condition consisted of three girls and three boys, resulting in a total sample size of six students for that analysis. The total sub-sample of students who provided consent across conditions included four girls in the library condition and three students in each of the remaining conditions (boys in the library, girls in the Web, and boys in the Web), resulting in a total sample size of 13 students.
} 
with Google, the documents that are "hit" or "returned" appear in a list along with a brief (typically one or two lines) description or excerpt from the linked document. The list can contain a few documents or hundreds of documents. The list is set up to present a maximum of 10 of these linked document excerpts per page.

The second type of behavior we are able to capture is scanning of the list of document excerpts that have been returned by a given Google query. Students could either scroll through and scan an entire list, or they could limit themselves to scanning a portion of the returned list. Based on our field notes, we are able to count how many of these document excerpts each student scanned, and to analyze these brief excerpts to see whether the documents excerpts that were scanned by students contained any information relevant to either the target-specific knowledge or the target-related knowledge questions.

The third and final type of information-seeking behavior we were able to index was browsing. Browsing consists of instances in which a student accessed (actually opened a document by clicking on the document link) and read or skimmed at least a portion of a given document on the list returned by the search keywords. Although it was not possible to capture exactly what document portions were read and or understood by students, we were able to assess the informational relevance of each browsed document. Specifically, each of the browsed documents was scored in terms of the amount of target-specific and target-related information provided. This provided us with an index of the quality of the learning resources used by each student.

\section{Searching}

Students' searching behavior was assessed by exploring the number of unique keywords or phrases they entered to query the database. There was a significant difference on this behavior $(F(1,13)=10.889, p=.006)$. Specifically, boys in the Web condition $(M=6.14)$ submitted a greater number of unique queries on average than girls $(M=2.14$; see Figure 3$)$. Submitting a greater number of keywords may account for the boys' superior gains in answering the target-specific as well as the target-related knowledge. That is, boys may have produced more significant learning opportunities based on the results of the searches they submitted. While the total number of documents hit or returned by the total number of searches submitted by each student did not differ across gender, it is conceivable that this larger variety of queries could have provided a broader variety of information. Furthermore, it is possible that boys and girls differed from one another in terms of how they pursued this information.

\section{Scanning and Browsing}

It is also possible that boys and girls differed from one another in terms of what they go on to do with the results returned by their Google queries. In order to 


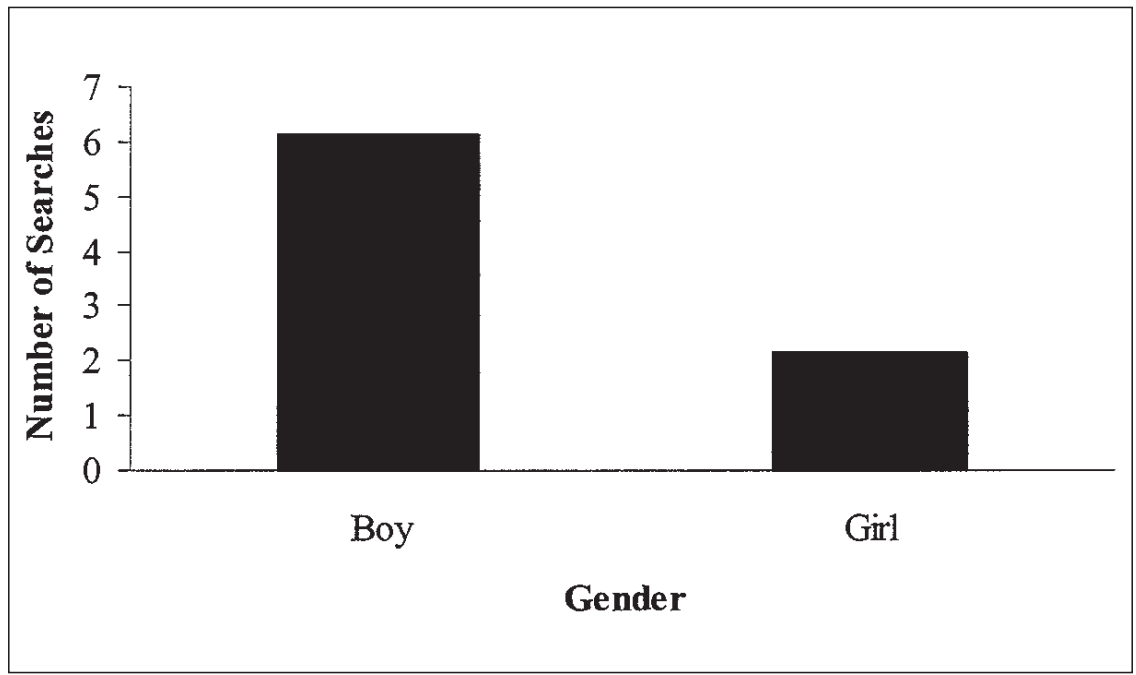

Figure 3. Number of unique searches submitted in Google by Gender.

investigate this possibility we used a MANOVA to compare the profiles of boys and girls behavior in terms of the number of document excerpts that they scanned and the number of documents that they browsed. The results of this analysis revealed a marginally significant difference in the profiles of boys and girls (multivariate $F(2,11)=3.79, p=.056)$. Basically, boys $(M=126.86)$ scanned more document excerpts than girls $(M=55.43$; see Figure $4 \mathrm{a})$, whereas girls $(M=23.43)$ browsed more documents than boys $(M=13.86$; see Figure $4 \mathrm{~b})$.

\section{Assessment of the Availability of Information in the Search Outcomes}

In order to further explore the possibility that the different patterns of scanning and browsing could explain the learning outcome differences between boys and girls, we also assessed the informational availability of the document excerpts or hits that these students scanned as well as the quality of the documents that they browsed. That is, one might make the argument that the boy's search results yielded higher quality document excerpts and/or documents than the girl's in the sense of greater availability of target-specific and target-related information. To assess this possibility, we tallied each instance of correct target-specific information and each instance of target-relevant information in the document excerpts that were scanned by students to yield two separate measures of the availability of information (one for target specific learning, and one for target 


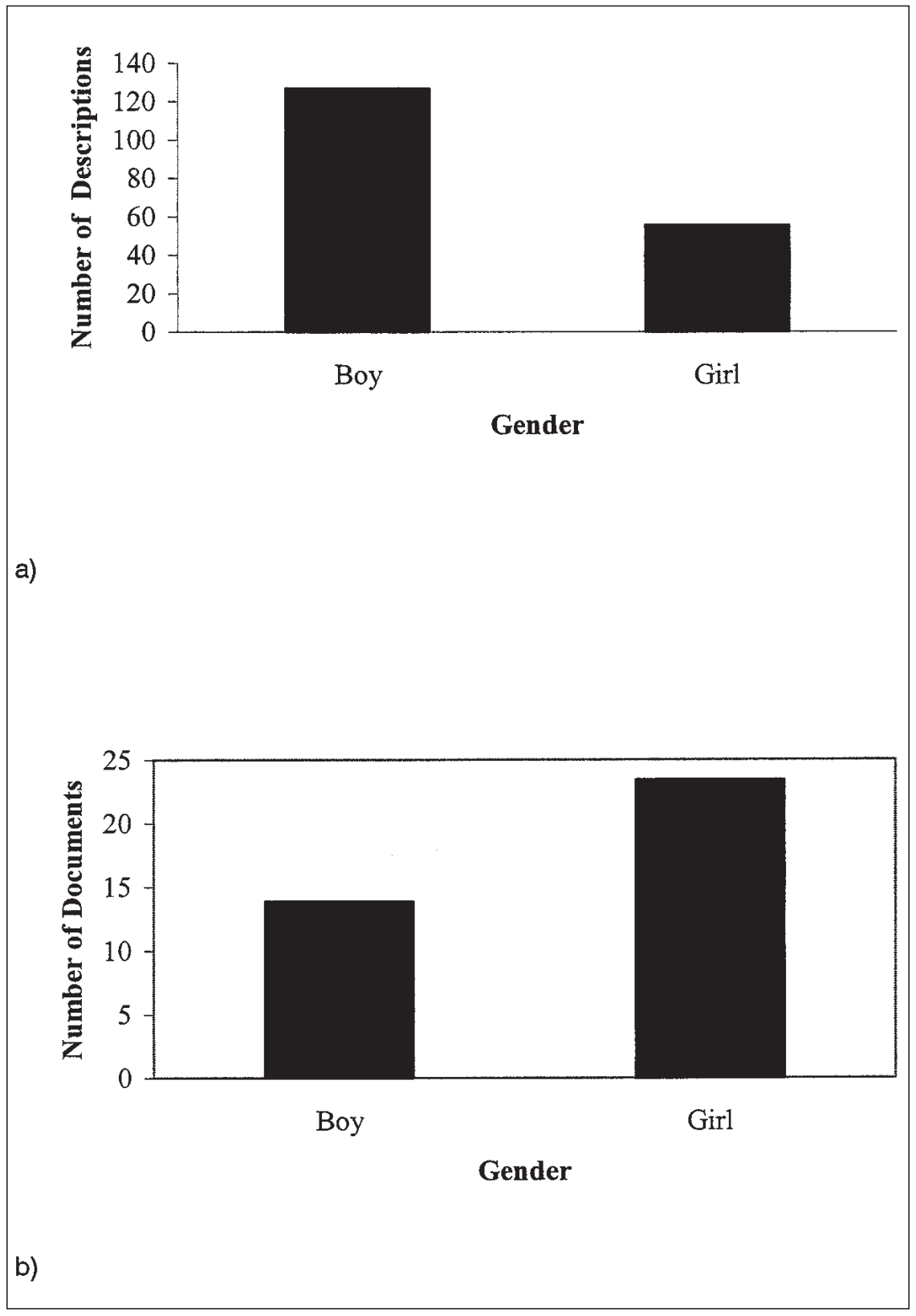

Figure 4. a) Number of Google excerpts scanned while searching on the Web by Gender. b) Number of documents browsed while searching on the Web by Gender. 
related learning). A similar method was used to generate two measures of the availability of information browsed by students. First we present the results of our analysis of the information availability for the document excerpts scanned, followed by an analysis of the actual documents browsed.

A MANOVA revealed a significant difference between the genders in terms of the availability of information in the documents excerpts scanned (multivariate $F(2,11)=4.96, p=.029)$. Basically, boys $(M=25.43)$ were exposed to more instances of document excerpts with target-specific information than girls $(M=10.14$; see Figure 5a), and more instances of target-related information $(M=9.29)$ than girls $(M=1.57$; see Figure $5 b)$. It should also be noted that both the amount of target-specific and target-related information available for each document description scanned remained higher for boys $(M=.202$ and $M=.07$, respectively) than for girls ( $M=.175$ and $M=.02$, respectively). The difference for the former (i.e, target-related information) was not statistically significant, however the latter (i.e., target-specific) continued to be statistically significant in favor of boys. Thus, boys and girls not only differed from one another in terms of the quantity of information that they scanned, they also differed in the quality of information they were exposed to during scanning. Specifically, boys scanned more documents than girls, and the documents that they scanned contained more task relevant information. These results also suggest that the search queries submitted by the boys were somewhat more accurate than those submitted by the girls.

Next, we assessed the quality of information that students browsed by coding and tallying the total number of correct target ideas, and the total number of correct incidental learning ideas contained in pages of the documents. A MANOVA of this data revealed no significant difference between genders in terms of the quality of information available in the documents browsed (multivariate $F(2,11)=0.91, p=.43)$. While boys $(M=11.43)$ were exposed to fewer instances of target-specific ideas in the documents they accessed than girls $(M=16.43$; see Figure 6a), and more instances of target-related information $(M=9.43)$ than girls $(M=7.86$; see Figure $6 \mathrm{~b})$, these differences were not sufficiently large to be reliably different.

\section{Differences between the Web and Library}

In this last section, we want to address potential differences in information seeking behavior that might account for the differences (although not statistically significant) in the size of learning gains between conditions. Recall that there was a greater overall gain in target-specific knowledge for the Web condition than for the Library condition (see Figure 1 again). Conversely, the knowledge scores for the target-related information were greater in the Library condition overall (see Figure 2 again). We believe that this pattern reflects variation in the kind of resources or documents that are provided by the Web and Library, and thus the 


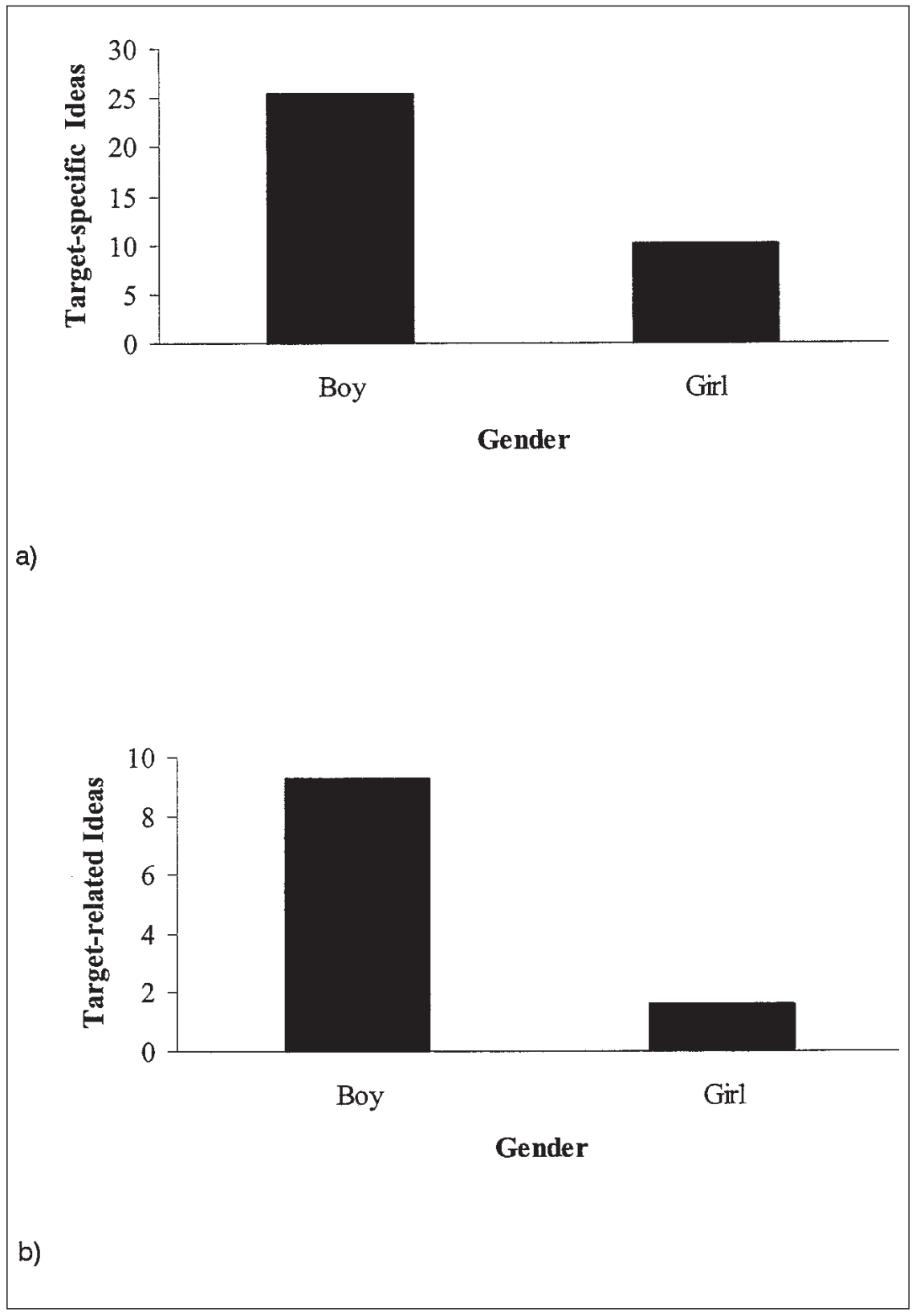

Figure 5. a) Number of target-specific hits while scanning document excerpts on the Web by Gender. b) Number of target-related hits while scanning document excerpts on the Web by Gender. 


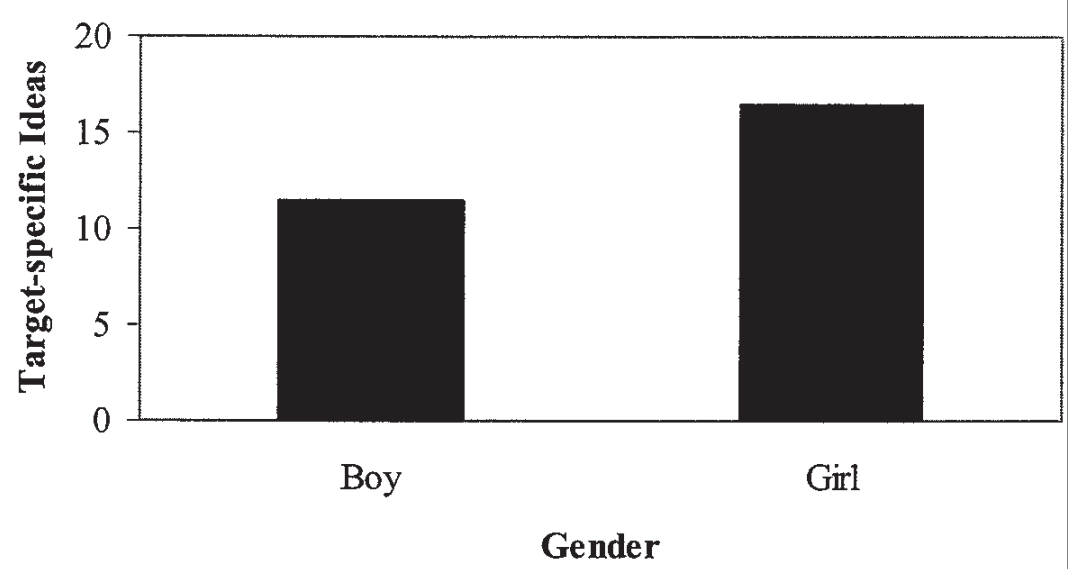

a)

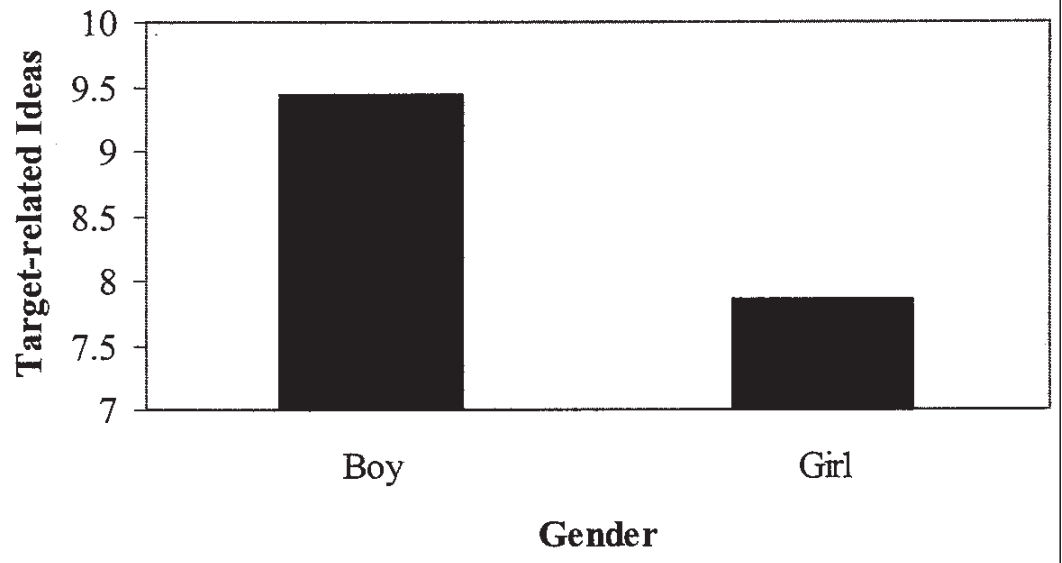

b)

Figure 6. a) Number of target-specific hits while browsing documents on the Web by Gender. b) Number of target-related hits while browsing documents on the Web by Gender. 
kind of learning opportunities each best supports. In the case of the Web, one tends to find short documents that are often very specific to the target information queried. This attribute may be particularly useful in situations where the user has a limited, well-defined search goal for very specific information (e.g., like the search task used in the current study). The Library resources, on the other hand, are primarily comprised of books that contain extensive background information that is relevant to multiple perspectives or questions that one could ask. This suggests that if one browses a Library book, one is likely to pick up a great deal of target-related information, but not necessarily the target-specific information. This pattern can be seen in Figures $7 \mathrm{a}$ and $7 \mathrm{~b}$. That is, Figure 7a shows the number of target-specific idea units found in information browsed by the students in the Web condition $(M=12.28)$ was significantly greater than the number of idea units found in the browsed documents in the Library condition $(M=3.57$; $F(1,24)=8.39, p=.009)$. The converse is true for the number of incidental information units contained in the documents. Figure $7 \mathrm{~b}$ shows that the number of incidental learning answers found in information browsed by the students was far greater in the Library condition $(M=22.73)$ than the Web condition $(M=8.88$; $F(1,24)=6.37, p=.02)$. Thus, the results are consistent with our assumption that resources such as a book-length document, contained a greater number of references to ideas probed in the incidental learning task than short documents in the Web condition. Such differences may account for the greater overall learning of incidental knowledge in the Library condition (see Figure 2 again).

\section{DISCUSSION 3}

The primary purpose of this study was to compare middle school students in terms of their ability to search for, browse, and learn information in response to a specific well-defined prompt using either new on-line technology (i.e., the Web), or more traditional off-line methods (i.e., the school Library). Specifically, we were interested in determining whether the Web offers any specific advantages to students over more traditional means when students are asked to perform a simple search task. In addition, we were interested in ascertaining whether middle school girls and boys perform similarly under the two search conditions. This is an important issue to address since some previous studies have suggested that girls may lag behind boys in the degree to which they are experienced with and motivated by new technology.

\footnotetext{
${ }^{3}$ In discussing our findings, the authors wish to caution the reader against making any gross generalizations given that random sampling procedures were not employed in the current study. Further replication of the results is necessary before any strong generalizations of the current findings can be made.
} 


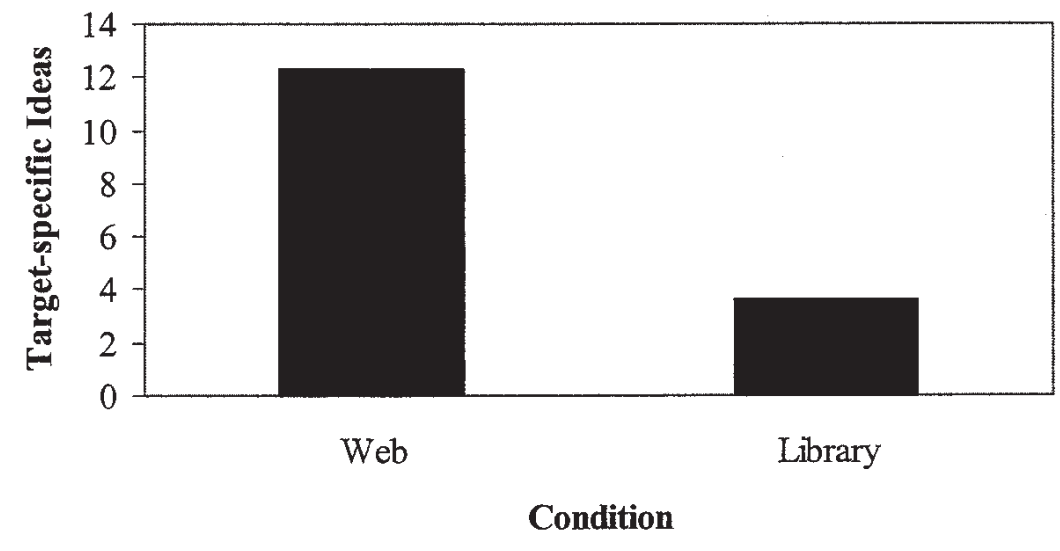

a)

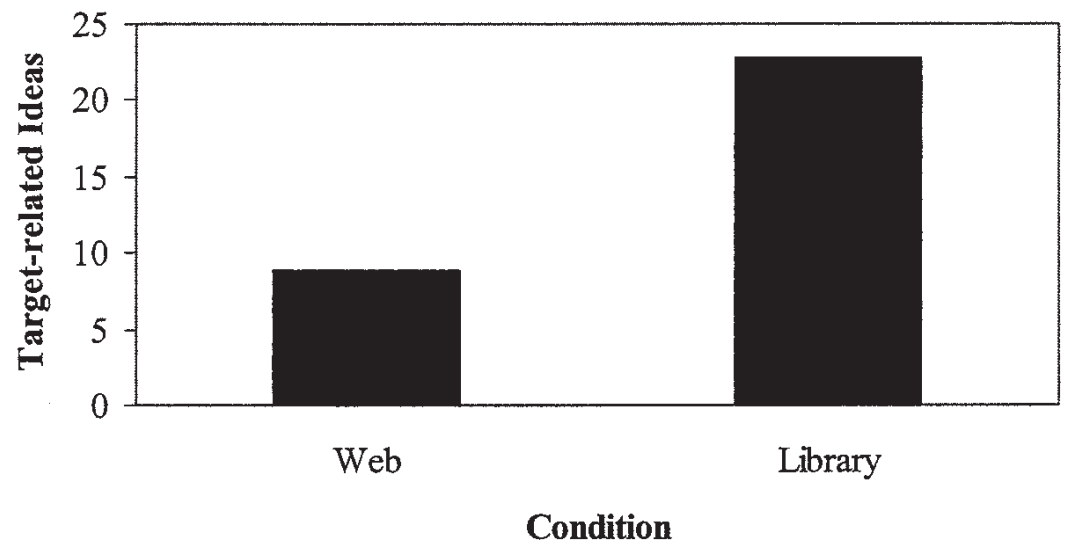

b)

Figure 7. a) Number of target-specific ideas hit while browsing documents by Condition. b) Number of target-related ideas hit while browsing documents by Condition. 


\section{Learning Outcomes}

The first issue we addressed was whether there were differences in learning outcomes between the two search groups. An encouraging result from this research is that students in both search conditions were able to make significant target knowledge gains. Thus, the middle school students that we studied were successful at using both the physical Library resources and the electronic resources provided by Web browsers and the Internet. This being said, however, there was a difference between boys and girls in terms of how much they learned when searching for information on the Web. Specifically, we found that boys learned significantly more target-specific and target-related information than girls. This is consistent with the findings reported in previous research (Light et al., 2000). Unlike previous research which has linked these differences to either ability or cross gender variation in attitudes toward technology, however, we were able to link these learning differences to variation in how boys and girls approached the search task in the Web environment. Others have also observed differences in how boys and girls approach open-ended questions. For example, one study (Moreno $\&$ Mayer, 1999) indicates that simply manipulating contextual characteristics of open-ended problem-solving questions, like wording, can elicit different response strategies from boys and girls. Therefore, the idea that boys and girls may respond somewhat differently to open-ended questions in the Web search context does not seem far fetched. But what characteristic(s) of the Web environment seem to be responsible for eliciting the gender difference in the current study?

Ironically, one potential advantage of using the Web to search for information may also be one of its drawbacks. That is, searching the Web with one or more queries typically returns a vast number of documents that the user must then sort through to filter out the specific information that they seek. Thus, using the Web to find information may produce many resources, however, the ability to use these resources successfully and efficiently may require extra or different navigation strategies on the part of the user than when using the more traditional Library. In particular, searching on the Web may require the user to have some efficient method to filter out which documents are most likely to be relevant to the current search goal(s) from a large pool of potentially relevant documents.

We observed that boys and girls each had a distinct approach to finding and selecting information in the Web condition. Boys had a tendency to scan many more document excerpts than girls, while girls had a tendency to actually open and browse the entire linked documents without going through a preliminary scanning step. We believe that it is this pattern of variability that was responsible for the different learning outcomes. In particular, this difference in search style may have been advantageous to boys in three ways. First, boys may have had more opportunities to come across the specific information being sought by directly locating it (or fragments of it) in the document excerpts. Furthermore, because document excerpts are much shorter than the average linked document, boys were 
able to scan many more document excerpts within the allotted time compared with the number of documents that girls were able to browse. Finally, the documents that boys did scan tended to contain a greater amount of information that was both specifically related and tangentially related to the search goal. The data indicate that all three of these potential advantages were indeed operating. Boys were exposed to many more relevant learning opportunities while scanning than girls were. Such scanning was apparently sufficient to pick up target-specific information relevant to addressing the assigned question and target-related information relevant to answering questions about the target topic in general. Thus boys were able to take direct advantage of the tendency for Web searches to produce many hits.

Girls on the other hand may have been somewhat penalized by the information quantity they encountered in the Web condition. Girls appeared to try to find the target information by actually opening and reading the individual documents rather than by attempting to filter through the document pool first. This strategy is inefficient and costly, particularly when one is faced with having to filter through so many potentially relevant information sources. Presumably however, girls could be taught to adopt the strategy used by boys in a fairly straightforward manner, allowing them to enjoy similar learning benefits to that experienced by boys. This possibility should definitely be followed up in a future training study. It is poignant to note that although the girls performance was poorer than boys in the Web condition, girls actually reported using the Web for academic purposes more often than boys. This finding clearly underscores the need to uncover the underlying cause(s) of the observed performance discrepancy and of finding ways to ameliorate it.

The current study demonstrates that, while middle school students can be successful at searching for, locating, reading, and learning specific information using either their local Library or the Web; the Web may offer certain advantages to students who know how to take advantage of distinct features offered by the Web. In particular, the Web seems to offer brief excerpts from the documents returned by a particular query. These one or two line excerpts can be scanned by the user to filter, and in a cursory way, to cover a large pool of documents in a very efficient manner. Our findings indicate that boys are able to take advantage of this characteristic. It should be pointed out that our findings also indicate that simply providing students with access to new technologies does not in and of itself guarantee increased learning outcomes. That is, the girls that participated in the Web condition in this study probably could have benefited from some simple training in how to use document excerpts as learning resources for this simple search task.

\section{Differences between the Web and the Library}

A second main finding of the current study has to do with the observation that the Library and the Web each support a different pattern of information filtering 
behavior that is independent of gender. This difference probably stems from the fact that, on the Web, it is very easy to move between the listing of search results to the electronic documents. All it takes is the click of a button. This is in contrast to dealing with books in the Library where one moves between search results, to actually finding the correct shelf, locating the document, looking in a table of contents, and flipping to the appropriate pages. It is easy to see how, in this second situation, one may be more likely to come across (and browse) information that is tangentially rather than directly related to the target of the search. That is, each of these steps present opportunities for learning information related to the specific target information.

Such differences suggest that the Web may be a more efficient means of searching for a limited well-defined set of information, but that the Library may provide richer exposure to related contextual information in addition to specific targeted facts. It would be interesting to investigate whether results similar to those reported here would occur in a study in which students were given a prompt to find extensive information that required a more elaborate response. In such a situation one might expect the Library condition to be more advantageous. This may be a particularly important research question to pursue given the current shift away from curriculums focused on textbook-based learning to those that place increasing emphasis on more open-ended discovery-based learning goals. Educators will need to know if and how the new technologies being incorporated into the schools will support these newer and more complex learning goals. Furthermore, if it is indeed the case that the Library provides unique advantages for searching for more elaborated information, such findings would provide a strong rationale for keeping traditional Libraries in our schools in addition to providing access to new information technologies like the Web.

\section{REFERENCES}

Andriessen, J., \& Sandberg, J. (1999). Where is education heading and how about A.I.? International Journal of Artificial Intelligence in Education, 10, 130-150.

Bilal, D. (1999). Web Search Engines for Children: A Comparative Study and Performance Evaluation of Yahooligans!, Ask Jeeves for Kids, and Super Snooper. Proceedings of the 62nd ASIS annual meeting (pp. 70-82). Washington, D.C., October 31-November 4, 1999.

Bilal, D. (2000). Children's use of the Yahooligans! Web search engine: I. Cognitive, physical, and affective behaviors on fact-based search tasks. Journal of the American Society for Information Science, 51(7), 646-665.

Camp, T. (1997). The incredible shrinking pipeline. Communications of the ACM, 40(10), 103-110.

Fidel, R., Davies, R. K., Douglass, M. H., Holder, J. K., Hopkins, C. J., Kushner, E. J., Miyagishima, B. K., \& Toney, C. D. (1999). A visit to the information mall: Web searching behavior of high school students. Journal of the American Society for Information Science, 50(1), 24-37. 
Goldman, S. R. (1996). Reading, writing, and learning in hypermedia environments. In H. van Osstendorp \& S. de Mul (Eds.), Cognitive aspects of electronic text processing (pp. 7-42). Norwood, NJ: Ablex.

Kadijevich, D. (2000). Gender differences in computer attitude among ninth-grade students. Journal of Educational Computing Research, 22(2), 145-154.

Large, A., \& Beheshti, J. (2000). The Web as a classroom resource: Reactions from the users. Journal of the American Society for Information Science, 51(12), 1069-1080.

Light, P., Littleton, K., Bale, S., Joiner, R., \& Messer, D. (2000). Gender and social comparison effects in computer-based problem solving. Learning and Instruction, 10, 483-496.

Linn, M. C., \& Hyde, J. S. (1989). Gender, mathematics, and science. Educational Researcher, 18(8), 17-19, 22-27.

Mitra, A., Steffensmeier, T., Lenzmeier, S., Avon, R., Qu, N., \& Hazen, M. (2000). Gender and computer use in an academic institution: Report from a longitudinal study. Journal of Educational Computing Research, 23(1), 67-84.

Moreno, R., \& Mayer, R. E. (1999). Gender differences in responding to open-ended problem-solving questions. Learning and Individual Differences, 11(4), 355-364.

Nachmais, R., Mioduser, D., \& Shemla, A. (2001). Information and communication technologies usage by students in an Israeli high school: Equity, gender, and inside/outside school learning issues. Education and Information Technologies, 6(1), 43-53.

Nahl, D. (1998). Learning the Internet and the structure of information behavior. Journal of the American Society for Information Science, 49(11), 1017-1023.

Owston, R. D. (1997). The World Wide Web: A technology to enhance teaching and learning? Educational Researcher, 26(2), 27-33.

Pelavin Research Institute (1997). Investing in school technology: Strategies to meet the funding challenge. Report prepared for the U.S. Department of Education. Available on-line http://www.ed.gov/pubs/techinvest.

Schacter, J., Chung, G. K. W. K, \& Dorr, A. (1998). Children's Internet searching on complex problems: Performance and process analyses. Journal of the American Society for Information Science, 49(9), 840-849.

Schumacher, P., \& Morahan-Martin, J. (2001). Gender, Internet and computer attitudes and experiences. Computers in Human Behavior, 17(1), 95-110.

Todman, J., \& Dick, G. (1993). Primary children and teachers' attitudes to computers. Computers in Education, 20(2), 199-203.

U.S. Department of Education (1997). The U.S. Department of Education Strategic Plan, 1998-2002. Available on-line at http://www.ed.gov/pubs/StratPln/.

Direct reprint requests to:
Dr. Marguerite Roy
e-mail: mar982@pitt.edu
Dr. Michelene Chi
e-mail: Chi@pitt.edu

3939 O’Hara Street

Learning Research and Development Center

University of Pittsburgh

Pittsburgh, PA 15260 